\title{
Completeness of Noncompact Analytic Spaces
}

\author{
By \\ Takeo OHSAWA*
}

\begin{abstract}
Let $X$ be a reduced paracompact complex analytic space of dimension $n$. It is proved that if $X$ has no compact irreducible branches of dimension $n$, then $X$ is $n$-complete, and if $X$ has finitely many compact irreducible branches of dimension $n, X$ is $n$-pseudoconvex, both in the sense of Andreotti-Grauert. Applying Andreotti-Grauert's finiteness theorem (resp. Barlet's theorem on the Steinness of cycle spaces) we deduce from our result Siu's theorem on analytic sheaf cohomology groups of noncompact complex spaces (resp. the Steinness of the space of $(n-1)$-cycles of analytic spaces whose $n$-dimensional irreducible branches are non-compact).
\end{abstract}

\section{§1. Introduction}

In the theory of complex analysis, it has long been known that for any noncompact Riemann surface Cousin's first problem is always solvable. In 1955, Malgrange [6] showed that for any paracompact noncompact complex manifold $X$ of dimension $n$, and for any locally free analytic sheaf $E$ over $X, H^{n}(X, E)$, the $n$-th cohomology group of $X$ with coefficients in $E$, vanishes. The latter result may be regarded as a natural generalization of the classical result. It was later refined by Komatsu [5], and finally completed by Siu [8], [9] in the following form:

Theorem S. Let $X$ be a (not necessarily reduced) paracompact complex analytic space of dimension $n$, and $\mathscr{F}$ a coherent analytic sheaf over $X$. Then,

1) $H^{n}(X, \mathscr{F})=0$, if every $n$-dimensional irreducible branch of $X$ is noncompact.

2) $\operatorname{dim} H^{n}(X, \mathscr{F})<\infty$, if there are only finitely many $n$-dimensional compact irreducible branches of $X$.

Received June 25, 1983.

Supported by Alexander von Humboldt-Foundation.

* Research Institute for Mathematical Sciences, Kyoto University, Kyoto 606, Japan. 
Siu's proof is based on the results of Malgrange and Komatsu, and carried out by means of standard techniques of sheaf theory. The purpose of the present note is to give a different proof of Theorem $\mathrm{S}$ by establishing the following

Theorem 1. Let $X$ be a reduced paracompact complex analytic space of dimension $n$. Then,

1) $X$ is $n$-complete, if every $n$-dimensional irreducible branch of $X$ is noncompact.

2) $X$ is strongly $n$-pseudoconvex, if there are only finitely many ndimensional compact irreducible branches of $X$.

Here, ' $n$-complete' and ' $n$-pseudoconvex' are in the sense of Andreotti-Grauert [1]. Theorem S follows immediately from Theorem 1 and Andreotti-Grauert's 'théorème 14' and its corollary in [1]. As a consequence we obtain the following

Theorem 2. Let $X$ be a paracompact $n$-dimensional complex analytic space and let $C_{q}(X)$ be the reduced analytic space of compact cycles of pure dimension $q$ of $X$ (cf. [2]). Then, $C_{n-1}(X)$ is a Stein space, if every $n$-dimensional irreducble branch of $X$ is noncompact.

For nonsingular $X$, Theorem 1 has been already proved by Greene-Wu [4] as a corollary of the proper harmonic embedding theorem for a complete Riemannian manifold (cf. [4], Corollary).

The author expresses his hearty thanks to Professors K. Fritzsche and M. Buchner who told him Greene-Wu's result and convinced him of the non-triviality of Theorem 1 on the way from Bonn to Göttingen. He also thanks Professor D. Barlet, who kindly sent him the article [3], which is closely related to the present paper.

\section{§ 2. Preliminaries}

In what follows we denote by $X$ a paracompact reduced complex analytic space of dimension $n$. Let $R(X)$ be the set of regular points of $X$ whose neighbourhoods have dimension $n$. We set $S(X):=X-$ 
$R(X)$. Then $S(X)$ is an analytic subset of $X$ with $\operatorname{dim} S(X)<n$. (cf. [7]). A real valued function $f$ on $X$ is said to be of class $C^{\infty}$ if, for any point $x \in X$, there exist a neighbourhood $U$, a holomorphic embedding $\iota: U \rightarrow \boldsymbol{C}^{N}$ for some $N \gg 0$, and a function $\tilde{f}$ of class $C^{\infty}$ on $\boldsymbol{C}^{N}$ such that $\iota^{*} \tilde{f}=\left.f\right|_{U}$. We shall denote $d f=0$ at $x$ if $d \tilde{f}=0$ at $\iota(x)$. This notion is clearly independent of the choice of $\iota$ and $\tilde{f}$. A function $f$ of class $C^{\infty}$ on $X$ is said to be $q$-convex (weakly $q$-convex) at $x$, if we can choose above $\tilde{f}$ so that $\partial \bar{\partial} \tilde{f}$ has at least $N-q+1$ positive (resp. nonnegative) eigenvalues at $\iota(x) . f$ is called $q$-convex (weakly $q$-convex) on $X$ if $f$ is $q$-convex (resp. weakly $q$-convex) at any point of $X . f$ is said to be exhausting if, for any $c \in \mathbb{R}, X_{f, c}:=\{x \in X ; f(x)<c\}$ is relatively compact in $X . \quad X$ is said to be $q$-pseudoconvex if there exists an exhaustion function $f: X \rightarrow \boldsymbol{R}$ of class $C^{\infty}$ and a compact subset $K \subset X$ such that $\left.f\right|_{X-K}$ is $q$-convex. $X$ is called $q$-complete if we can choose $f$ so that $K=\emptyset$.

Lemma. Let $Y \subset X$ be an analytic subspace of dimension $<n$. Then, there exists an n-convex function $\phi_{Y}$ defined on a neighbourhood of $Y$ such that $\left.\phi_{Y}\right|_{Y}$ is exhausting.

Proof. If $\operatorname{dim} Y=0$, then the conclusion is clear. Assume that the proposition is true for any analytic subspace $Y \subset X$ with $\operatorname{dim} Y$ $<r<n$. Let $\operatorname{dim} Y=r$. Then, by the induction assumption, there exists an $n$-convex function $\phi_{S(Y)}$ on an open neighbourhood $V$ of $S(Y)$ in $X$, such that $\left.\phi_{S(Y)}\right|_{S(Y)}$ is exhausting. Let $W$ be a neighbourhood of $S(Y)$ in $X$ such that $\bar{W} \subset V$, and let $\rho: X \rightarrow \mathbb{R}$ be a function of class $C^{\infty}$ satisfying $\left.\rho\right|_{W}=1$ and $\left.\rho\right|_{X-V}=0$. Let $\mathscr{U}=\left\{U_{i}\right\}(i=1,2, \ldots)$ be a locally finite system of open sets of $X-S(Y)$ such that $\bigcup_{i=1}^{\infty} U_{i}=$ $X-S(Y)$ and for any $U_{i} \in \mathscr{U}$, there exists a function $\phi_{i}: U_{i} \rightarrow \boldsymbol{R}$ of class $C^{\infty}$ such that

$$
\left.\phi_{i}\right|_{U_{i} \cap Y}=0,\left.\quad\left(d \phi_{i}\right)\right|_{U_{i} \cap Y}=0 .
$$

$\phi_{i}$ is weakly l-convex at any point $x \in U_{i} \cap Y$.

The existence of such $\mathscr{U}$ and $\left\{\phi_{\imath}\right\}$ is assured by the fact that $Y-S(Y)$ is nonsingular and of dimension $r^{*}$. Let $\left\{\rho_{\imath}\right\}$ be a partition of unity

\footnotetext{
* The argument used here is the same as D. Barlet's proof of $(n+1)$-completeness of neighbourhoods of a compact analytic subset of dimension $n$ (cf. Séminaire F. Norguet 1979, Lecture Notes no. 807 Springer Verlag).
} 
associated to $\mathscr{U}$ satisfying $0 \leqq \rho_{i} \leqq 1$ for any $i$. Let $\chi: X \rightarrow \boldsymbol{R}$ be a function of class $C^{\infty}$ such that $\chi(x) \geqq 0$ for any $x \in X, \chi(x)=1$ outside $W$, and $\chi(x)=0$ on a neighbourhood of $S(Y)$. Clearly, for sufficiently large numbers $N$ and $N_{i} \gg N(i=1,2, \ldots)$.

$$
\phi_{Y}^{\prime}:=N \rho \exp \phi_{S(Y)}+\sum_{i=1}^{\infty} N_{i} \chi \rho_{i} \phi_{i}
$$

is $n$-convex on a neighbourhood of $Y$. Let $\psi$ be any exhausting function of class $C^{\infty}$ on $X$. Then replacing $N$ and $N_{i}$ by larger numbers if necessary, we can make $\phi_{Y}:=\phi_{Y}^{\prime}+\phi$ an $n$-convex function on a neighbourhood of $Y$. Clearly, $\left.\phi_{Y}\right|_{Y}$ is exhausting. q.e.d.

From now on we assume that every $n$-dimensional irreducible branch of $X$ is noncompact. By the Lemma, there exists an $n$-convex function $\phi_{S(X)}$ on a neighbourhood of $S(X)$ such that $\left.\phi_{S(X)}\right|_{S(X)}$ is exhausting. Hence there exist an open neighbourhood $\Omega$ of $S(X)$ and an exhausting function $\phi: X \rightarrow \boldsymbol{R}$ of class $C^{\infty}$ such that $\left.\phi\right|_{\bar{\Omega}}=\left.\phi_{S(X)}\right|_{\bar{\Omega}}$. In what follows we put $X_{c}:=X_{\phi, c}$. By Sard's theorem there exists an increasing sequence of real numbers $c_{1}, c_{2}, \ldots$, such that

$$
\begin{aligned}
& \lim _{\nu \rightarrow \infty} c_{\nu}=\infty, \\
& d \phi \text { is nowhere zero on } \partial X_{c_{\nu}}-S(X),
\end{aligned}
$$

where $\partial X_{c_{\nu}}$ denotes the boundary of $X_{c_{\nu}}$ in $X$. We choose positive numbers $\varepsilon_{\nu}, \nu=1,2, \ldots$ so that $c_{\nu-1}+\varepsilon_{\nu-1}<c_{\nu}-\varepsilon_{\nu}$ and $d \phi$ is nowhere zero on $X_{c_{\nu}+\varepsilon_{\nu}}-X_{c_{\nu}-\varepsilon_{\nu}}-\Omega$.

We fix a hermitian metric $d s^{2}$ on $X-S(X)$ such that the sum of the eigenvalues of $\partial \bar{\partial} \phi$ with respect to $d s^{2}$, which shall be denoted by trace $\partial \bar{\partial} \phi$, is everywhere positive on $\Omega-S(X)$ (cf. [10] $\$ 12$ Lemma 4.1.). For any $c \in \boldsymbol{R}$, let $Y_{c}$ be the union of the connected components of $X-X_{c}-S(X)$ whose closures in $X$ are compact. We choose a subsequence $\left\{c_{\nu_{\alpha}}\right\},(\alpha=1,2, \ldots)$ of $\left\{c_{\nu}\right\}$ as follows. We set $\nu_{1}=1$. Suppose that $c_{\nu_{\alpha}}$ have already been determined. Since every $n$ dimensional irreducible branch of $X$ is noncompact, $\phi$ is bounded on $Y_{c_{\nu_{\alpha}}}$. Hence there exists an integer $\tilde{\nu}$ such that $c_{\tilde{D}}>\sup _{Y_{c_{\nu}}} \phi$. We put $c_{\nu_{\alpha+1}}=c_{\tilde{\nu}}$. For simplicity we put $c_{\alpha}:=c_{\nu_{\alpha}}$ and $\varepsilon_{\alpha}:=\varepsilon_{\nu_{\alpha}}$.

A function $\lambda:(-\infty, c) \rightarrow \boldsymbol{R}(c \in \boldsymbol{R} \cup\{\infty\})$ is called a convex increasing function if $\lambda$ is of class $C^{\infty}, \lambda^{\prime}(t)>0$, and $\lambda^{\prime \prime}(t) \geqq 0$ on $(-\infty, c)$. 
Proposition. Let the notations be as above. Then, for $\alpha=1,2, \ldots$, there exist open neighbourhoods $\Omega_{\alpha}$ of $S(X)$ satisfying $\Omega \supset \bar{\Omega}_{1} \supset \bar{\Omega}_{2} \supset \ldots \supset$ $\bar{\Omega}_{\alpha} \supset \ldots$, nonnegative functions $\Phi_{\alpha}$ of class $C^{\infty}$ on $X$, positive numbers $\delta_{\alpha}$ with $2 \delta_{\alpha}<\varepsilon_{\alpha}$, and convex increasing functions $\lambda_{\alpha}: \boldsymbol{R} \rightarrow \boldsymbol{R}$ such that

$$
\Phi_{\alpha}=\lambda_{\alpha}(\phi) \quad \text { on } \Omega_{\alpha} \cap X_{c_{\alpha-2}-\delta_{\alpha-2}} \cup\left(X-X_{c_{\alpha}}\right) \text {, }
$$

(8) $\operatorname{trace} \partial \bar{\partial} \Phi_{\alpha}>0$ on $X_{c_{\alpha}}-X_{c_{\alpha-2}-\delta_{\alpha-2}}-Y_{c_{\alpha-2}}-S(X)$ where we put $c_{-1}=c_{0}=-\infty$ and $\delta_{-1}=\delta_{0}=0$,

(9) $\quad X_{c_{\alpha-2}}-\bar{X}_{c_{\alpha-2}-2 \delta_{\alpha-2}}-\Omega_{\alpha}$ is diffeomorphic to $\left(\partial X_{c_{\alpha-2}}-\Omega_{\alpha}\right) \times \mathbb{R}$ as manifolds with boundary.

Proof. Let $\alpha \geqq 1$ and assume that $\Omega_{i}, \Phi_{i}, \delta_{i}$ and $\lambda_{i}$ are obtained for $0 \leqq i \leqq \alpha-1$. Here we define $\Omega_{0}:=\Omega, \Phi_{0}:=\phi$, and $\lambda_{0}(t):=t$. Let $x \in X_{c_{\alpha}}-\Omega-Y_{c_{\alpha-2}}-X_{c_{\alpha-2}}$ be any point. Then, there exists an injective $C^{\infty}$ map of maximal rank

$$
r:(-1,1] \times \boldsymbol{R}^{2 n-1} \rightarrow \bar{X}_{c_{\alpha}}-\bar{X}_{c_{\alpha-2}-\varepsilon_{\alpha-2}}-\bar{Y}_{c_{\alpha-2}}-S(X)
$$

such that

$$
\begin{aligned}
& \gamma((0,0, \ldots, 0))=x \\
& \gamma\left(\{1\} \times \boldsymbol{R}^{2 n-1}\right) \subset \partial X_{c_{\alpha}} \\
& \gamma\left(\{0\} \times \boldsymbol{R}^{2 n-1}\right) \subset X_{c_{\alpha}}-X_{c_{\alpha-2}} \\
& \gamma\left(\{0\} \times \boldsymbol{R}^{2 n-1}\right) \subset \partial X_{c_{\alpha-2}} \text { if } x \in \partial X_{c_{\alpha-2}}
\end{aligned}
$$

We set $T(\gamma, r, s):=\gamma\left(\left[-1+\frac{1}{s}, 1\right] \times B(r)\right)$, where $B(r):=\left\{\left(x_{1}, \ldots\right.\right.$, $\left.\left.x_{2 n-1}\right) ; \sum_{i=1}^{2 n-1} x_{i}^{2} \leqq r\right\}$ and $s>0$. For any $r>0$, the union of the interior points of $T(\gamma, r, 1)$ for all possible $\gamma$ contains $\bar{X}_{c_{\alpha}}-\Omega-Y_{c_{\alpha-2}}-X_{c_{\alpha-2}}$ which is compact since $\phi$ is exhausting. Hence there exist finitely many $\gamma_{1}, \ldots, \gamma_{m_{\alpha}}$ so that

$$
\bigcup_{i=1}^{m_{\alpha}} T\left(\gamma_{i}, 1,1\right) \supset \bar{X}_{c_{\alpha}}-\Omega-Y_{c_{\alpha-2}}-X_{c_{\alpha-2}} .
$$

Let $\Omega_{\alpha}$ be a neighbourhood of $S(X)$ with smooth boundary such that

$$
\begin{aligned}
& \Omega_{\alpha} \cap \bigcup_{i=1}^{m_{\alpha}} T\left(\gamma_{i}, 3,3\right)=\emptyset, \\
& \partial \Omega_{\alpha} \text { intersects } \partial X_{c_{\alpha-2}} \text { transversally, } \\
& \bar{\Omega}_{\alpha} \subset \Omega_{\alpha-1} .
\end{aligned}
$$




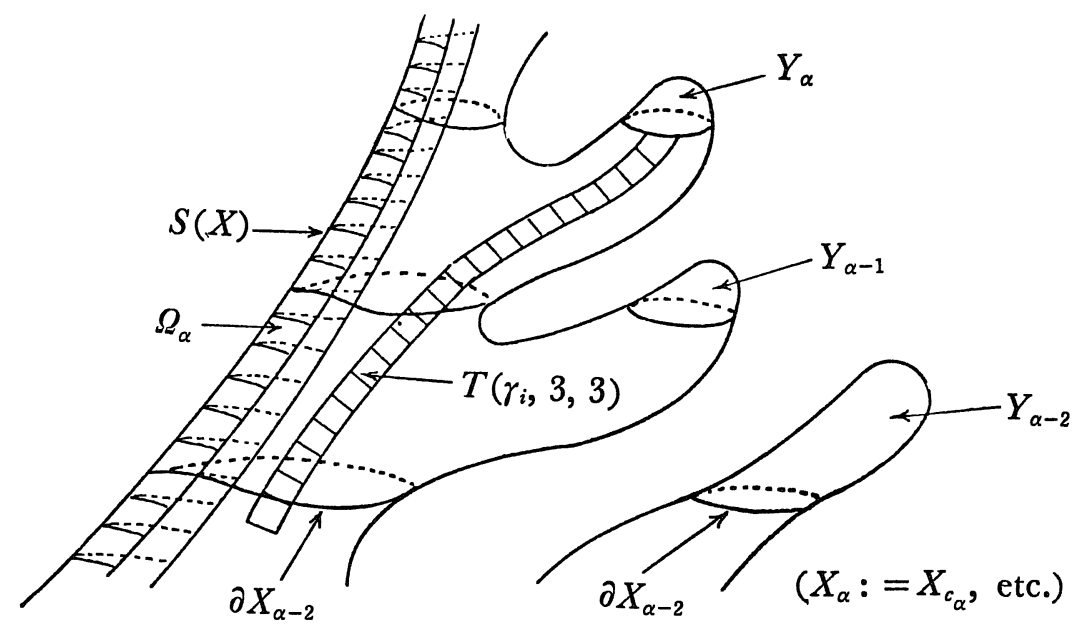

Figure

Let $\varepsilon_{\alpha}^{\prime}$ be a positive number such that $d \phi$ is nowhere zero on $\bar{X}_{c_{\alpha}}-X_{c_{\alpha}-\varepsilon_{\alpha}^{\prime}}-\Omega_{\alpha}$, and let $\delta_{\alpha-2}$ (if $\alpha \geqq 3$ ) be a positive number such that $2 \delta_{\alpha-2}<\varepsilon_{\alpha-2}$ and $\left(\partial X_{c_{\alpha-2}}-\Omega_{\alpha}\right) \times \boldsymbol{R}$ is diffeomorphic to $X_{c_{\alpha-2}}$ $\bar{X}_{c_{\alpha-2}-2 \delta_{\alpha-2}}-\Omega_{\alpha}$. We fix an $s_{0}$ so that $1<s_{0}<3$ and

$$
T\left(\gamma_{i}, 3, s_{0}\right) \subset \bar{X}_{c_{\alpha}}-X_{c_{\alpha-2}-\delta_{\alpha-2}}-S(X), \text { for any } i=1, \ldots, m_{\alpha} .
$$

To each $\gamma_{i}$, we associate a diffeomorphism $F_{i}: X \rightarrow X$ such that

$$
\begin{aligned}
& \left.F_{i}\right|_{X-T\left(\gamma_{i}, 3, s_{0}\right)}=i d . \\
& F_{i}\left(T\left(\gamma_{i}, 2, \frac{2 s_{0}+1}{3}\right)\right) \subset \bar{X}_{c_{\alpha}}-X_{c_{\alpha}-\varepsilon_{\alpha}^{\prime}} \\
& F_{i}\left(T\left(\gamma_{i}, 1, \frac{s_{0}+2}{3}\right)\right) \subset \bar{X}_{c_{\alpha}}-X_{c_{\alpha}-\varepsilon_{\alpha}^{\prime} / 2} .
\end{aligned}
$$

The existence of such diffeomorphism $F_{i}$ is clear from the elementary theory of ordinary differential equations. We set

$$
L_{\alpha}:=\frac{m_{\alpha}\left(\max _{i} \sup _{X_{c_{\alpha}}-\Omega_{\alpha}}\left|\operatorname{trace} \partial \bar{\partial} F_{i}^{*} \phi\right|\right)}{\min _{i} \inf _{F_{i}^{-1}\left(X_{c_{\alpha}}-X_{c_{\alpha}-\varepsilon_{\alpha}^{\prime}}\right)-\Omega_{\alpha}}\left(\operatorname{length} \partial F_{i}^{*} \phi\right)^{2}}
$$

where the length is measured by $d s^{2}$. Then, at any point $x \in \bigcup_{i=1}^{m_{\alpha}} T\left(\gamma_{i}, 2, \frac{2 s_{0}+1}{3}\right)$, we have

$$
\operatorname{trace} \partial \bar{\partial}\left(\sum_{i=1}^{m_{\alpha}} \exp L_{\alpha} F_{i}^{*} \phi\right)>0 .
$$


We choose $M_{\alpha} \gg L_{\alpha}$ so that at any point $x \in\left(\left(\bar{X}_{c_{\alpha}}-X_{c_{\alpha}-\varepsilon_{\alpha}}\right) \cup\left(\bar{X}_{c_{\alpha-2}}-\right.\right.$ $\left.\left.X_{c_{\alpha-2}-\varepsilon_{\alpha-2}}\right) \cup \bar{\Omega}\right)-S(X)$,

$$
\operatorname{trace} \partial \bar{\partial} \exp M_{\alpha} \phi>0
$$

and

$$
\operatorname{trace} \partial \bar{\partial}\left(\exp M_{\alpha} \phi+\sum_{i=0}^{m_{\alpha}} \exp L_{\alpha} F_{\imath}^{*} \phi\right)>0 .
$$

Let $\xi_{\mu}: \boldsymbol{R} \rightarrow \boldsymbol{R}$ be a convex increasing function defined by

$$
\xi_{\mu}(t)=t+\mu \exp \left(-\frac{1}{\left(t-\exp L_{\alpha}\left(c_{\alpha}-\varepsilon_{\alpha}^{\prime}\right)\right)^{2}}+t-\exp L_{\alpha}\left(c_{\alpha}-\varepsilon_{\alpha}^{\prime}\right)\right) .
$$

Since $\left(\xi_{\mu}\left(\exp L_{\alpha} t\right)\right)^{\prime \prime} \geqq L_{\alpha}\left(\xi_{\mu}\left(\exp L_{\alpha} t\right)\right)^{\prime}$ for any $\mu \geqq 0$, we have

$$
\begin{aligned}
& \operatorname{trace} \partial \bar{\partial}\left(\exp M_{\alpha} \phi+\sum_{i=1}^{m_{\alpha}} \xi_{\mu}\left(\exp L_{\alpha} F_{\imath}^{*} \phi\right)\right)>0 \\
& \text { for any } \mu \geqq 0, \\
& \text { on }\left(\left(\bar{X}_{c_{\alpha}}-X_{c_{\alpha}-\varepsilon_{\alpha}}\right) \cup\left(\bar{X}_{c_{\alpha-2}}-X_{c_{\alpha-2}-\varepsilon_{\alpha-2}}\right) \cup \bar{\Omega}\right)-S(X) .
\end{aligned}
$$

Therefore, by (15) and (22), for sufficiently large $\mu_{\alpha}$,

$$
\begin{aligned}
& \operatorname{trace} \partial \bar{\partial}\left(\exp M_{\alpha} \phi+\sum_{i=1}^{m_{\alpha}} \xi_{\mu_{\alpha}}\left(\exp L_{\alpha} F_{\imath}^{*} \phi\right)\right)>0, \\
& \text { on } \bar{X}_{c_{\alpha}}-Y_{c_{\alpha-2}}-X_{c_{\alpha-2} \varepsilon_{\alpha-2}}-S(X) .
\end{aligned}
$$

We set

$$
\Phi_{\alpha}=\exp M_{\alpha} \phi+\sum_{i=1}^{m_{\alpha}} \xi_{\mu_{\alpha}}\left(\exp L_{\alpha} F_{i}^{*} \phi\right) .
$$

Clearly, $\Omega_{\alpha}, \Phi_{\alpha}, \delta_{\alpha-2}$, and $\lambda_{\alpha}:=\exp M_{\alpha} t+\sum_{i=1}^{m_{\alpha}} \xi_{\mu_{\alpha}}\left(\exp L_{\alpha} t\right)$ satisfy all the requirements.

q. e. d.

\section{§3. Proof of Theorem}

Let the notations be as in section 2. We choose $\Omega_{\alpha}, \Phi_{\alpha}, \delta_{\alpha-2}$, and $\lambda_{\alpha}$ as in the proof of the Proposition. Inductively, we construct exhausting functions $\Psi_{\alpha}: X_{c_{\alpha}} \rightarrow \boldsymbol{R}$ of class $C^{\infty}$ satisfying the following properties :

$\left(B_{\alpha}\right)$

$$
\begin{aligned}
& \Psi_{\alpha}=\Psi_{\alpha-1} \text { on } X_{c_{\alpha-2}-2 \delta_{\alpha-2}}, \\
& \Psi_{\alpha}>\alpha \text { on } X_{c_{\alpha}}-X_{c_{\alpha-2}}, \\
& \Psi_{\alpha} \text { is } n \text {-convex on } X_{c_{\alpha}},
\end{aligned}
$$

$\left(D_{\alpha}\right) \quad \Psi_{\alpha}=p_{\alpha}(\phi)$ on $X_{c_{\alpha}} \cap \Omega_{\alpha}$ for some convex increasing function $p_{\alpha}$. 
We put

$$
\Psi_{1}=\frac{1}{c_{1}^{\prime}-\Phi_{1}}+1
$$

where $c_{1}^{\prime}=\lambda_{1}\left(c_{1}\right)$. Then $\left(A_{1}\right) \sim\left(D_{1}\right)$ are satisfied by $\Psi_{1}$.

Assume that we have already $\Psi_{i}$ for $i<\alpha$ which satisfy $\left(A_{i}\right) \sim$ $\left(D_{i}\right)$. Let $N_{\alpha}$ be an integer such that

$$
\Psi_{\alpha-1}^{-1}\left(\left(-\infty, N_{\alpha}\right)\right) \supset \bar{Y}_{c_{\alpha-2}} \cup \bar{X}_{c_{\alpha-2}},
$$

and let $\rho_{\alpha}: \boldsymbol{R} \rightarrow \boldsymbol{R}$ be a function of class $C^{\infty}$ such that $\rho_{\alpha}(t)=1$ for $t \leqq N_{\alpha}$ and $\rho_{\alpha}(t)=0$ for $t \geqq N_{\alpha}+1$. We put

$$
\widetilde{\Psi}_{\alpha-1}=\left\{\begin{array}{ccc}
\rho_{\alpha}\left(\Psi_{\alpha-1}\right) & \text { on } & X_{c_{\alpha-1}} \\
0 & \text { on } & X-X_{c_{\alpha-1}}
\end{array}\right.
$$

Let $\eta:\left(-\infty, \lambda_{\alpha}\left(c_{\alpha}\right)\right) \rightarrow \boldsymbol{R}$ be a function of class $C^{\infty}$ such that

$$
\begin{aligned}
& \eta(t)=0 \text { for } t \leqq \lambda_{\alpha}\left(c_{\alpha-2}-2 \delta_{\alpha-2}\right) \\
& \eta^{\prime}(t)>0, \eta^{\prime \prime}(t)>0, \text { for } \lambda_{\alpha}\left(c_{\alpha-2}-2 \delta_{\alpha-2}\right)<t<\lambda_{\alpha}\left(c_{\alpha}\right) \\
& \lim _{t \rightarrow \lambda_{\alpha}\left(c_{\alpha}\right)} \eta(t)=\infty
\end{aligned}
$$

and

$$
\left(\eta \circ \lambda_{\alpha}\right)^{\prime \prime}(t)-\left(\eta \circ \lambda_{\alpha}\right)^{\prime}(t)\left(\rho_{\alpha} \circ p_{\alpha-1}\right)^{\prime}(t)>0, \text { for } \quad c_{\alpha-2}-\delta_{\alpha-2} \leqq t<c_{\alpha} .
$$

The existence of such a function is obvious. By (7) and $\left(D_{\alpha-1}\right)$ there exist functions $\sigma_{\alpha}$ and $\tau_{\alpha}$ such that

$$
\eta\left(\Phi_{\alpha}\right)=\sigma_{\alpha}\left(\Psi_{\alpha-1}\right)=\tau_{\alpha}(\phi) \quad \text { on } \quad \Omega_{\alpha} \cap X_{c_{\alpha}} .
$$

By (37), $\sigma_{\alpha}^{\prime \prime}(t)>0$ on $\left[p_{\alpha-1}\left(c_{\alpha-2}-\delta_{\alpha-2}\right), N_{\alpha}\right]$. Clearly $\sigma_{\alpha}^{\prime}(t)>0$ on $\left[p_{\alpha-1}\left(c_{\alpha-2}-\delta_{\alpha-2}\right), N_{\alpha}\right]$ and $\sigma_{\alpha}(t)=0$ on $\left(-\infty, p_{\alpha-1}\left(c_{\alpha-2}-2 \delta_{\alpha-2}\right)\right]$. Let $Z_{\alpha-2}$ be the union of the connected components of $X_{c_{\alpha-2}}-\bar{X}_{c_{\alpha-2}-2 \delta_{\alpha-2}}$ $-\Omega_{\alpha}$ whose closures intersect $\bar{Y}_{c_{\alpha-2}}$. Note that $Z_{\alpha-2}$ is diffeomorphic to $\left(\partial Y_{c_{\alpha-2}}-\Omega_{\alpha}\right) \times \boldsymbol{R}$. We put

$$
\Psi_{\alpha \kappa}=\left\{\begin{array}{l}
\widetilde{\Psi}_{\alpha-1}+\kappa \eta\left(\Phi_{\alpha}\right) \quad \text { on } X_{c_{\alpha}}-Y_{c_{\alpha-2}}-Z_{\alpha-2} \\
\Psi_{\alpha-1}+\kappa \sigma_{\alpha}\left(\Psi_{\alpha-1}\right) \text { on } Z_{\alpha-2} \cup Y_{c_{\alpha-2}},
\end{array}\right.
$$

where $\kappa$ denotes a positive number. Then, by (7), (9), and (34), $\Psi_{\alpha, \kappa}$ is a well defined exhausting function of class $C^{\infty}$ on $X_{c_{\alpha}}$. By $\left(B_{\alpha-1}\right),\left(C_{\alpha-1}\right)$, and (35), for sufficiently large $\kappa_{\alpha}, \Psi_{\alpha, \kappa_{\alpha}}$ is $n$-convex on $X_{c_{\alpha}}$ and $\Psi_{\alpha, \kappa_{\alpha}}>\alpha$ on $X_{c_{\alpha}}-X_{c_{\alpha-2^{*}}}$ We put $\Psi_{\alpha}:=\Psi_{\alpha, \kappa_{\alpha}}$. Then $\Psi_{\alpha}$ satisfies $\left(A_{\alpha}\right) \sim\left(D_{\alpha}\right)$. 
To prove the $n$-completeness of $X$, we define

$$
\Psi=\Psi_{\alpha} \quad \text { on } \quad X_{c_{\alpha-1}-2 \delta_{\alpha-1}} \text { for any } \alpha \geqq 1 .
$$

By $\left(A_{\alpha}\right) \sim\left(C_{\alpha}\right), \Psi$ is a well defined $n$-convex exhausting function on $X$. Therefore $X$ is $n$-complete.

If there are only finitely many $n$-dimensional compact irreducible branches of $X$, let $X^{\prime}$ be the union of the $n$-dimensional noncompact irreducible branches and the lower dimensional branches. Then, there exists an $n$-convex exhausting function $\psi^{\prime}$ on $X^{\prime}$. Let $A$ be the union of $n$-dimensional compact branches of $X$. Let $\beta=\sup _{A \cap X^{\prime}} \psi^{\prime}+1$. We set

$$
\dot{\varphi}=\left\{\begin{array}{lr}
\exp \left(-\frac{1}{\left(\psi^{\prime}-\beta\right)^{2}}+\psi^{\prime}-\beta\right) & \text { on } X^{\prime} . \\
0 & \text { on } A
\end{array}\right.
$$

Then, $\psi$ is an exhausting function of class $C^{\infty}$ on $X$ which is $n$-convex outside a compact subset of $X$. Thus $X$ is $n$-pseudoconvex. q. e. d.

\section{§. Applications}

The following theorem has been obtained by Barlet:

Theorem B (cf. [3] Théorème 4. Note that the definition of $n^{-}$ completeness is different from ours.) Let $X$ be a finite dimensional ncomplete analytic space. Then, $C_{n-1}(X)$, the reduced analytic space of compact cycles of pure dimension $n$ of $X$, is Stein.

Hence, combining Theorem B with Theorem 1, we obtain Theorem 2.

The following theorem was obtained by Andreotti and Grauert:

Theorem A-G (cf. [1] Théorèm 14 and its corollary). Let $X$ be a (not necessarily reduced) complex analytic space, and $\mathscr{F}$ a coherent analytic sheaf over $X$. Then,

1) $\quad H^{i}(X, \mathscr{F})=0$ for $i \geqq q$, if $X$ is q-complete.

2) $\operatorname{dim} H^{i}(X, \mathscr{F})<\infty$ for $i \geqq q$, if $X$ is $q$-pseudoconvex.

Hence, combining Theorem A-G with Theorem 1, we obtain Theorem S. 


\section{References}

[1] Andreotti, A. and Grauert, H., Théorème de finitude pour la cohomologie des espaces complexes, Bull. Soc. Math. France 90 (1962), 193-259.

[2] Barlet, D., Espace analytique réduit des cycles analytiques complexes compacts d'un espace analytique complexe de dimension finite, Fonctions de plusieurs variables complexes, II, Séminaire Francois Norguet, 1974/75, p-1-158, Berlin, Springer-Verlag, 1975 (Lecture Notes in Mathematics, 482) (Thèse Sc. math. Université Paris VII, 1975).

[3] - Convexité de l'espace de cycles, Bull. Soc. math. France, 106 (1978), 373-397.

[4] Greene, R. E. and Wu, H., Embedding of open Riemannian Manifolds by harmonic functions, Ann. Inst. Fourier, Grenoble 25, 1 (1975), 215-235.

[5] Komatsu, H., Resolutions by hyperfunctions of sheaves of solutions of differential equations with constant coefficients, Math. Ann. 176 (1968), 77-86.

[6] Malgrange, B., Existence et approximation des solutions des équations aux dérivées partielles et des équation de convolution, Ann. Inst. Fourier, Grenoble 6 (1955-1956), 271-355.

[7] Narashimhan, R., Introduction to the theory of analytic spaces, Lecture Notes 25, SpringerVerlag, 1966.

[8] Siu, Y, T., Analytic sheaf cohomology groups of dimension $n$ of $n$-dimensional noncompact complex manifolds, Pacific J. Math. 28 (1969), 407-411.

[9] Analytic sheaf cohomology groups of dimension $n$ of $n$-dimensional complex spaces, Trans. Amer. Math. Soc. 143 (1969), 77-94.

[10] Vesentini, E., Lectures on Levi Convexity of Complex Manifolds and Cohomology Vanishing Theorems, Tata Inst. Bombay 1967. 